Man and Nature

L'homme et la nature

\title{
Voltaire revu et corrigé par la Révolution Française : Le cas de La mort de César
}

\section{Muriel Usandivaras-Mili}

Volume 10, 1991

URI : https://id.erudit.org/iderudit/1012636ar

DOI : https://doi.org/10.7202/1012636ar

Aller au sommaire du numéro

Éditeur(s)

Canadian Society for Eighteenth-Century Studies / Société canadienne d'étude du dix-huitième siècle

ISSN

0824-3298 (imprimé)

1927-8810 (numérique)

Découvrir la revue

Citer cet article

Usandivaras-Mili, M. (1991). Voltaire revu et corrigé par la Révolution

Française : Le cas de La mort de César. Man and Nature / L'homme et la nature, 10, 209-225. https://doi.org/10.7202/1012636ar

Copyright (c) Canadian Society for Eighteenth-Century Studies / Sociéte canadienne d'étude du dix-huitième siècle, 1991
Ce document est protégé par la loi sur le droit d'auteur. L'utilisation des services d’Érudit (y compris la reproduction) est assujettie à sa politique d'utilisation que vous pouvez consulter en ligne.

https://apropos.erudit.org/fr/usagers/politique-dutilisation/ 


\section{Voltaire revu et corrigé par la Révolution Française: Le cas de La mort de César}

Il n'est personne qui en sortant de la représentation de Brutus ou de La mort de César ne soit disposé à poignarder le scélérat qui tenterait d'asservir son pays. Je demande que le Comité de Salut Public prenne des mesures pour qu'on ne joue que des pièces républicaines (le député Delacroix, Le Moniteur, 15 août 1793).

Dans le projet révolutionnaire, $l$ 'idée de 'régénération' domine très tôt la scène politique et artistique. Cette 'régénération' ou 'nouvelle naissance' (l'un des sens du mot dans L'Encyclopédie qui le classe dans le vocabulaire religieux) impliquait pour être efficace la destruction de tous les symboles qui de près ou de loin pouvaient rappeler l'Ancien Régime. C'est ce qu'on a appelé le 'Vandalisme révolutionnaire' et sur lequel il convient $\mathrm{d}^{\prime}$ être assez nuancé. ${ }^{1}$

Un an à peine après le début de la Révolution, le décret du 20 juin 1790 déclarait que 'la dignité d'un peuple libre' est incompatible avec des monuments qui rappellent 'des idées d'esclavage.' Il s'agissait donc $d^{\prime}$ en ôter tout ce qui pouvait être un symbole de la monarchie absolue (par exemple les statues représentant Louis XIV). Lors de la séance du 11 août 1792, un député de l'Assemblée législative déclara:

Il faut enfin déraciner tous les préjugés royaux. Je demande que l'Assemblée prouve au peuple qu'elle s'occupe de sa liberté, et que la statue de la Liberté soit élevée sur les mêmes piédestaux. ${ }^{2}$ (c'est nous qui soulignons)

Dès lors,tous les signes extérieurs qui rappelèrent la royauté furent détruits: destruction des tombeaux de St-Denis, supression des armoiries sur les grilles, frontons, tombes, statues, reliures...etc.

Cette tentative de 'purge' s'appliqua aussi aux textes et aux auteurs de théâtre. Le langage et les personnages ${ }^{3}$ devaient être modifiés pour ne plus comporter de mentions de l'Ancien Régime. Ainsi furent sup- 
primés les titres de noblesse attribués à un personnage ou mentionnés dans le dialogue, l'usage de 'monsieur' et 'madame,' le vouvoiement, les formules de politesse ou de bienséance...etc. ${ }^{5}$ Quant aux auteurs, ils prenaient des pseudonymes, qui pouvaient cacher leur origine de petite noblesse provinciale. ${ }^{6}$

Le décret du 2 août 1793 rendit ces mesures obligatoires pour le texte des pièces:

Tout théâtre sur lequel seront représentées des pièces tendant à dépraver l'esprit public et à réveiller la honteuse superstition de la royauté, sera fermé et les directeurs arrêtés et punis selon la rigueur des lois. (souligné par nous)

Ces mesures s'appliquèrent non seulement aux créations mais aussi aux reprises de textes classiques, ceux de Corneille, Racine ${ }^{7}$ et Molière, mais aussi ceux de Voltaire furent passés au peigne fin afin d'en extirper tous les signes de corruption et de dépravation. Cette épuration allait de la simple substitution de $\operatorname{mots}^{8}$ à la récriture partielle ou totale des pièces.

Une récriture en particulier a retenu notre attention. La mort de César de Voltaire ${ }^{9}$ jouée pour la première fois en 1743, tint l'affiche pendant 7 représentations et en eut une cinquantaine ${ }^{10}$ pendant les dix années de la révolution. Gohier, qui fut chargé de la récrire, en donna une version nouvelle entre novembre 1793 et le 9 thermidor an II , date à laquelle elle devait être définitivement abandonnée pour retrouver celle originale de Voltaire.

Il est bon de s'interroger d'abord sur la place de La mort de César dans l'oeuvre dramatique de Voltaire avant de s'arrêter à l'analyse du texte lui-même. Seront ensuite examinées la nature et l'étendue des modifications qui ont été apportées au texte: peut-être expliqueront-elles les raisons politiques et/ou esthétiques qui les auront suscités?

Conçue lors du séjour forcé de Voltaire en Angleterre (1726), La mort de César était achevée en 1731 ( $c^{\prime}$ est la 5e tragédie écrite par Voltaire). Représentée à l'Hotel de Sassenage en 1733 puis au collège d'Harcourt en 1735, elle finit par affronter les feux de la rampe du Théâtre-Français le 29 août 1743 après avoir été interdite du 10 juin au 29 août.

C'est une tragédie en 3 actes dont le sujet a été emprunté au Jules César de Shakespeare (1599). Ce n'est pas une traduction mais bien plutôt une assez libre adaptation comme l'écrit Voltaire lui-même:

Nous donnons cette édition de la tragédie de La mort de César de Voltaire, et nous pouvons dire qu'il est le premier qui ait fait connaitre les muses anglaises en France. (...)Parmi les morceaux les plus singuliers des poètes anglais que notre ami nous traduisit, il nous donna la scène $\mathrm{d}^{\prime}$ Antoine et du peuple romain, prise 
de la tragédie de Jules César, écrite il y a cent cinquante ans par le fameux Shakespeare, et jouée encore aujourd'hui avec un très grand concours sur le théâtre de Londres. Nous le priâmes de nous donner le reste de la pièce; mais il était impossible de la traduire.

Shakespeare était un grand génie, mais il vivait dans un siècle grossier; et l'on retrouve dans ses pièces la grossièreté de ce temps, beaucoup plus que le génie de l'auteur. Voltaire, au lieu de traduire l'ouvrage monstrueux de Shakespeare, composa dans le goût anglais, ce Jules César, que nous donnons au public. ${ }^{11}$

De semblables idées sur le théâtre anglais, Voltaire en avait déjà exprimé dans son Discours sur la Tragédie, préface à Brutus, tragédie en 5 actes, conçue elle aussi en Angleterre et représentée pour la première fois le 11 décembre 1730 .

Il a manqué jusqu'à présent à presque tous les auteurs tragiques de votre nation cette pureté, cette conduite régulière, ces bienséances de l'action et du style, cette élégance, et toutes ces finesses de l'art qui ont établi la réputation du théâtre français depuis le grand Corneille. ${ }^{12}$ ( $c^{\prime}$ est nous qui soulignons)

Voltaire reprendra presque les mêmes mots plus tard pour parler des tragiques anglais dans ses Lettres philosophiques (1734). Dans sa Lettre XVIII, Sur la Tragédie, il tire en effet,après sa traduction du monologue d'Hamlet et d'un passage de Dryden, la conclusion suivante:

C'est dans ces morceaux détachés ques les tragiques anglais ont jusqu'ici excellé: leurs pièces presque toutes barbares, dépourvues de bienséance, d'ordre, de vraisemblance, ont des lueurs étonnantes au milieu de cette nuit. ${ }^{13}$ ( $c^{\prime}$ est nous qui soulignons)

Composées à la même époque et imitées des tragiques anglais, Brutus et La mort de César ont toutes deux un personnage nommé Brutus, protagoniste dans la première, antagoniste dans la seconde:

La mort de César est le pendant de Brutus. Dans Brutus, Voltaire a montré le vieux Romain immolant ses enfants à la liberté; dans La mort de César, il montre l'autre Brutus immolant son père à la République. ${ }^{14}$

Evidemment, il ne s'agit pas du même personnage! Dans la première, est mis en scène Lucius Junius Brutus qui vécut au VIe siècle av. J.C. et qui fut le premier consul de Rome avec Valérius Publicola (présent dans 
la pièce) après l'abolition de la royauté et l'exil de la famille royale des Tarquins. Il présida lui-même à l'éxécution de ses deux fils accusés d'avoir voulu comploter le retour de la royauté. Quant à 'l'autre Brutus,' il faut voir en lui un personnage attesté par l'histoire, né vers 85 , mort en 42 av.J.C. Il fut élevé par Caton et reconnu par César dans le complot auquel il a effectivement participé activement; obligé de fuir après l'assassinat de César, et se voyant vaincu, il se suicida.

Pour compléter cette mise en scène de l'histoire romaine, il importe d'ajouter Rome sauvée ou Catilina représentée pour la première fois le 24 février 1752 et publiée l'année suivante (Voltaire a alors presque 60 ans). L'avertissement mis en tête de la pièce dans l'édition Kehl des Oeuvres complètes de Voltaire indique non seulement les rapprochements entre cette pièce et $L a$ mort de César mais aussi le but de telles pièces, les réactions qu'elles peuvent susciter chez les spectateurs:

Cette pièce, ainsi que La mort de César, est d'un genre particulier, le plus difficile de tous peut-être, mais aussi le plus utile....Elles [ces pièces] ont surtout l'avantage précieux de donner à l'âme de l'élévation et de la force: en sortant de ces pièces, on se trouve plus disposé à une action de courage, plus éloigné de ramper devant un homme accrédité, ou de plier devant le pouvoir injuste et absolu. ${ }^{15}$ (c'est nous qui soulignons)

Nous reviendrons plus tard sur les effets de cette pièce chez le spectateur, effets que probablement Voltaire n'avait pas prévus ou voulus sciemment pour nous arrêter à ce que, comme dramaturge, il pouvait penser de sa pièce La mort de César.

D'après Voltaire, cette tragédie apporte deux innovations au théâtre tragique français. Tout d'abord elle n'a que 3 actes au lieu des 5 habituels, ensuite elle ne comporte aucun rôle féminin et, par conséquent, aucune scène amoureuse. Cette diminution du nombre des actes, la lettre ${ }^{16}$ qui précède le texte de la pièce en rend compte. On y apprend que si Voltaire a choisi de faire durer l'action deux heures ( 3 actes) au lieu de trois ( 5 actes), c'est que d'après lui les Français ne peuvent rester plus longtemps à écouter des discussions politiques sur scène, eux qui sont accoutumés 'à voir soupirer Mithridate sur le point de marcher au Capitole. ${ }^{17}$ Mais de quoi sont faites les discussions politiques dans la pièce?

La scène se passe à Rome au Capitole dans les années 65 av.J.C.Une conversation entre Antoine et César nous apprend non seulement que le préteur Brutus est le plus farouche adversaire politique de ce dernier mais encore qu'il est son fils, fils d'une union tenue secrète. Nous assistons ensuite à une discussion entre les conjurés complotant la mort 
prochaine du dictateur dans le dessein de rétablir la république à Rome. Brutus, resté seul, a un entretien avec César: ce dernier lui apprend qu'il est son père et tente-après de longs rappels historiques sur la corruption de la république romaine et la nécessité de l'établissement d'un pouvoir fort-de le ramener dans son camp. Brutus refuse de céder sur ses convictions politiques.

Pris entre son amour pour son père et celui de la république, Brutus essaie de faire renoncer César au projet qu'il a de devenir roi et le prévient même d'un complot qui se fomente contre sa vie. César persiste. Alors, à la fin de la pièce, Brutus se joint aux meurtriers pour disparaître poursuivi par la vengeance du peuple romain.

La pièce est effectivement une suite de discours tour à tour favorables et hostiles au rétablissement de la république à Rome, Brutus incarnant l'idéal républicain face à César, qui fait valoir les mérites du despotisme. Pour l'un, la volonté populaire est souveraine, pour l'autre, il est nécessaire qu'elle soit contrôlée, chacun mettant à contribution l'histoire de Rome pour renforcer sa position.

Dans une lettre du 15 mars 1736 à M. de Lamarre, Voltaire dit de sa pièce que 'c' est ce combat singulier entre la tendresse et la fureur de la liberté qui, [seul pouvait] rendre la pièce intéressante. ${ }^{18}$

De 'ce combat singulier,' les personnages féminins sont écartés alors que d'après Voltaire, dans des tragédies traitant du même sujet, les auteurs rendent César et Brutus 'amoureux et jaloux' (préface de Voltaire à l'édition de 1736 de La mort de César). En réalité, Voltaire lui-même garde un personnage féminin dans son Brutus - Tullie, fille de Tarquin dont le fils de Brutus, Titus, est amoureux malgrè l'hostilité des familles entres elles. Dans La mort de César, pas de femme non plus que de confident: les personnages principaux sont constamment face à face sans tiers qui les écoute et qui puisse les amener à changer d'avis.

Le constat de la présence quasi-permanente de femmes dans la tragédie française: 'Corneille $n$ 'a fait aucune pièce sans amour' ${ }^{19}$ a été un sujet de réflexions pour Voltaire: 'personne n'ose guérir le théâtre français de cette contagion. ${ }^{20}$ Dans Les Lettres philosophiques, il fera la remarque suivante:

la coutume d'introduire de l'amour à tort et à travers dans les ouvrages dramatiques passa de Paris à Londres vers l'an 1660 avec nos rubans et nos perruques. Les femmes, qui parent les spectacles, comme ici, ne veulent plus souffrir qu'on leur parle d'autre chose que d'amour. ${ }^{21}$

Dans La mort de César, l'attention des spectateurs est tout entière fixée sur le conflit central sans distraction aucune, conflit qui comporte une 
exposition, une apogée et un dénouement, ce qui explique pourquoi Voltaire a choisi de faire sa tragédie en 3 actes et non en 5 .

Le premier acte révèle le conflit aux spectateurs: les deux hommes qui s'opposent le plus sur le plan politique sont ceux les plus proches par les liens du sang (le père et le fils). Ce premier acte tourne autour de César (il est présent dans les 4 scènes), c'est lui qui parle, dirige, convoque et renvoit. L'acte se termine par la question suivante: César révélerat-il à Brutus qu'il est son fils?

Le deuxième acte est consacré presque entièrement aux conjurés qui ont décidé la mort de César et dont Brutus fait figure de chef (il est présent dans les 5 scènes). Dans la dernière scène, il apprend de la bouche de César à la fois le secret de sa naissance et l'offre de partager l'empire avec lui. Sa réponse sera brève et nette:

Fais-moi mourir sur l'heure ou cesse de régner (II,V).

L'acte se termine sur l'échec de César pour résoudre le conflit. Au troisième acte, Brutus, après avoir appris aux conjurés le secret de sa naissance et réaffirmé qu'il ne manquerait pas à son devoir: ${ }^{22}$ 'Je ne trahirai point mon pays pour mon père' (III, II), tente dans un dernier et vain effort de résoudre le conflit, c'est-à-dire de convaincre César de renoncer au pouvoir suprême; celui-ci est assassiné et le peuple romain qui avait au préalable approuvé les meurtriers de César, finit-après un habile discours d'Antoine-par se retourner contre eux.

Le rideau tombe sur ces mots d'Antoine confiant au sénateur Dolabella ce qu'il pense du peuple romain:

Précipitons ce peuple inconstant et facile:

Entraînons-le à la guerre; et sans rien ménager,

Succédons à César, en courant le venger (III,VIII).

C'est ce texte qui a été représenté jusqu'en novembre 1793, date à laquelle, Gohier, alors ministre de la Justice, ${ }^{23}$ a apporté des modifications dont nous verrons maintenant la nature et l'étendue. Nous nous servirons à cette fin du texte de La mort de César pris dans les éditions Garnier des Oeuvres complètes de Voltaire, Théâtre, tome II, pages 296 à 366.

Quel est le contexte avant l'intervention de Gohier qui commence à la scène VII de l'acte III ?

Nous sommes à la fin de la pièce: le meurtre de César vient d'avoir lieu dans la scène prècédente où les spectateurs ont entendu les cris des conjurés poignardant César derrière le rideau: 
LES CONJURES, derrière le théâtre.

Meurs, expire, tyran! Courage Cassius!.

DOLABELLA

$\mathrm{Ah}$ ! courons le sauver. ${ }^{24}$

La scène VII commence avec l'entrée du sénateur Dolabella, des Romains et de l'un des conjurés, Cassius, 'un poignard à la main':

\section{CASSIUS}

C'en est fait, il n'est plus.

DOLABELLA

Peuples, secondez-moi,perçons ce traître.

CASSIUS

Peuples, imitez-moi, vous n'avez plus de maître. ${ }^{25}$

L'intervention de Gohier commence après ce premier vers de Cassius. D'abord, il fait parler deux sénateurs (faisant partie des conjurés) qui chez Voltaire n' intervenaient plus et que l'on avait entendus pour la dernière fois dans la scène II, Acte III où ils préparaient le complot avec leurs camarades. Decime et Cimber interviennent ici en effet pour appuyer Cassius contre Dolabella, qui, parlant en faveur de César, en appelle à la vengeance du peuple contre ses meutriers. Le mutisme du peuple ici fait contraste avec les quelques mots d'approbation que Voltaire lui prétait. ${ }^{26}$

Le travail de Gohier dans cette scène ne se borne pas à des modifications d'ordre terminologique, il retranche certains vers de Voltaire et en ajoute d'autres (les ajouts sont nécessaires par suite de l'adjonction de personnages parlants).

Ainsi, il supprime l'intervention presque immédiate de Dolabella pour laisser à Cassius une tirade inaugurale de 7 vers (repris de Voltaire).

Dolabella répond à Cassius en se présentant comme le champion prêt à défendre la mémoire de César. Cassius rétorque et après lui, Decime qui, armé d'un poignard (celui qui avait servi tantôt), va le brandir pour appuyer ses dires sur la nécessité de supprimer ceux qui prétendraient devenir roi. ${ }^{27}$

Ces 18 vers dits par Cassius, Dolabella et Decime expliquent au peuple en quoi le meurtre de César était devenu inéluctable: en affichant ses prétentions au pouvoir suprême, il était devenu un obstacle à la restauration de la république à Rome et la nécessité de sa supression ne faisait alors plus de doutes même pour ses partisans du début tels 
Cassius et Decime. ${ }^{28}$ Ils avaient donc éliminé celui qui prétendait devenir 'le tyran couronné.'

Voltaire terminait sa scène avec le serment que faisaient les Romains de soutenir Cassius face à Antoine dont l'éloquence était connue:

\section{CASSIUS}

Souvenez-vous, Romains, de ces serments sacrés.

\section{ROMAINS}

Aux vengeurs de l'Etat, nos coeurs sont assurés. ${ }^{29}$

Gohier supprime cette entente entre Cassius et les Romains pour faire dialoguer, Cimber, Dolabella et Cassius.

Voltaire, dans cette scène, ne donnait que deux vers à Dolabella, Gohier lui en accorde 31 et $1 / 2$ pour qu'il défende César et attaque ses assassins. Il les met aussi en garde contre la versatilité du peuple qui pourrrait bien se retourner contre ceux-là mêmes qu'il vient d'applaudir:

\section{DOLABELLA}

Ce géant à cent bras que tout succés enivre Pourra bien se lever, mais c'est pour te poursuivre

Trop souvent inquiet de sa propre grandeur,

Prodigue également $\mathrm{d}^{\prime}$ amour et de fureur, Inconstant dans ses gouts, ingrat, léger, frivole,

C'est pour la renverser qu'il se crée une idole,

Compte ses favoris trop tard désabusés. ${ }^{30}$

Enfin, et c'est là un point important, Gohier profite de cette scène pour éclaicir un point de terminologie: le sens du mot 'assassinat.' Pour Dolabella, c'est un crime; pour Cassius, c'est une delivrance.

\section{DOLABELLA}

Est-ce en assassinant que l'on défend ses droits?

CASSIUS

... l'homme courageux ....

Délivre son pays et $\mathrm{n}^{\prime}$ assassine pas. ${ }^{31}$

Cette discussion qui permet à chacun de donner des arguments prépare le peuple romain à écouter Antoine; Voltaire, au contraire, le laissait sans aucune préparation face à Antoine. 
La scène VIII de Voltaire commençait avec l'arrivée d'Antoine dont l'attitude était d'emblée ainsi commentée par les Romains:

\section{UN ROMAIN}

Mais Antoine parait.

AUTRE ROMAIN

\section{Qu'osera-t-il nous dire?}

\section{UN ROMAIN}

Ses yeux versent des pleurs, il se trouble, il soupire.

\section{UN AUTRE}

Il aimait trop César. ${ }^{32}$

Gohier supprime ces remarques pour, d'entrée de scène, laisser parler Cimber et Decime qui ne cachent pas leur hostilité vis-à-vis d'Antoine (les 4 premiers vers de la scène VIII). Il ne place pas non plus Antoine sur le podium, place de choix donnée par Voltaire. Enfin, il réduit considérablement la longueur et la portée de son discours.

Antoine se présentait comme celui qui, tout en pleurant la mort d'un ami, semble approuver ce qui vient de lui arriver:

Oui, je l'aimais, Romains; ...

Sans doute il fallait bien que César fut coupable. ${ }^{33}$

Puis il allait tenter de défendre la mémoire de César en faisant appel à sa générosité proverbiale envers le peuple, envers même ceux qui l'avaient assassiné et, parmi ceux-ci, Brutus, son fils. C'est à la révélation de ce lien de parenté entre l'assassin et la victime qu'Antoine constatait un changement chez ses auditeurs:

... je vois frémir vos généreux courages:

Amis, je vois les pleurs qui mouillent vos visages. ${ }^{34}$

Enfin, il annonçait, argument de poids, que si Brutus était le fils naturel de César, les Romains étaient 'ses enfants dans son coeur adoptés' et dignes d'être ses héritiers:

Brutus ou Cassius eût-il fait davantage?

ROMAINS

$\mathrm{Ah}$ ! nous les détestons. Ce doute nous outrage. ${ }^{35}$ 
Gohier ne garde pratiquement rien de l'éloquence persuasive d'Antoine. Ce dernier entre sur scène, reste de plain-pied avec les autres et énonce des faits déjà connus de tous:

Romains, César n'est plus ...

Il meurt assassiné. ${ }^{36}$

Et à ces mots, Antoine dévoile le cadavre de César aux yeux des Romains. Voltaire avait fait - si l'on peut dire - un meilleur usage scénique de ce cadavre: Antoine ne l'exposait aux regards des Romains qu'après s'être assuré de les avoir convaincus. De plus Antoine adoptait un comportement mélodramatique se jetant aux pieds du corps, effet supprimé par Voltaire qui nous montre Antoine, immobile, désignant du doigt le cadavre au peuple:

Affreux évènement, ô spectacle funeste!

Du plus grand des Romains, voilà ce qui vous reste. ${ }^{37}$

L'appel au soulèvement lancé ensuite par Antoine manque de force au point qu'il est obligé d'en venir rapidement à l'argument du lien de parenté entre Brutus et César, lequel n'émeut personne. A bout d'argument, il révèlera ( $c$ 'était le dernier argument utilisé par Voltaire) que l'héritage de César appartient aux Romains.

La fin de la scène VIII est prévisible que ce soit chez Voltaire ou chez Gohier. L'appel aux bons sentiments des Romains permettait à Antoine de désigner les assassins de César à la vindicte publique. Gohier, au contraire, en fait un coupable qui est accusé de vouloir prendre la place de César:

... de l'Etat en vain tu veux saisir les rênes

Et de tes faibles mains nous imposer des chaines:

Licteurs, qu'on le saisisse au nom du souverain. ${ }^{38}$

Antoine ne peut plus se défendre et les Romains passent du côté de Cassius dans un mouvement de scène qui évidement n' existait pas chez Voltaire. En effet, celui-ci, dans sa scène VIII et dernière, nous fait assister au triomphe d'Antoine et à l'annonce du châtiment qui est réservé aux meutriers de César.

Gohier ajoute deux scènes totalement inédites. Bien que quelques-uns de ses vers soient empruntés au texte de Voltaire, Il n'en demeure pas moins que Gohier fait ici un véritable travail d'auteur. 
La première scène supplémentaire, c'est-à-dire la scène IX, nous montre Cassius s'adressant aux Romains maintenant sans aucune opposition, Antoine et Dolabella venant d'être arrêtés dans la scène prècédente. Elle compte 38 vers dont 7 sont directement repris de la scène VII, acte III de Voltaire. Elle a pour fonction de dire aux Romains que leur tâche n'est pas achevée et que la seule mort de César ne suffit pas à supprimer définitivement la tyrannie. Le combat n'est pas terminé; il faut demeurer vigilant:

Vous aurez à combattre ...

Ne nous endormons point ...

Que de périls encore il vous faudra braver! $!^{39}$

La scène se termine par un discours de Cassius dont la force est soulignée par des impératifs réitérés:

Rappelons ... la paix ...

Etouffons des méchants les fureurs intestines,

Et de la liberté réparons les ruines.

Sachons apprécier le règne heureux des lois.

Prouvons que les Romains $n^{\prime}$ ont pas besoin des rois. ${ }^{40}$

Le dernier mot prononcé par Cassius est 'liberté,' et est immédiatement suivi d'un changement de décor avec, dans le fond du théâtre, l'émergence de la statue de la Liberté qui rassemble autour d'elle acteurs et même spectateurs. ${ }^{41}$

La deuxième scène supplémentaire, $c^{\prime}$ est-à-dire la scène $X$ et dernière, comprend 14 vers sans aucun emprunt à Voltaire. Elle permet à Brutus d'adresser une prière à la Liberté avant de succomber à ses pieds:

Ah! Que Rome soit libre et que Brutus expire. ${ }^{42}$

La scène s'achève par un serment des Romains:

Nous jurons d'imiter son courage hérö̈que:

Vive la liberté! Vive la République! ${ }^{43}$

Quel bilan peut-on faire de l'intervention de Gohier sur le texte de Voltaire et comment l'expliquer?

Des chiffres d'abord... Le texte de Voltaire comprend 1038 vers; la version de Gohier en compte 193, c'est à dire un peu plus de $16 \%$. De 
ceux-ci, il faut retrancher les vers de Voltaire repris (intégralement ou pas) par Gohier, c'est-à-dire 31 vers. Il reste donc à Gohier un total de 162 vers qui lui appartiennent, soit $15.6 \%$. On peut ici parler de récriture partielle. $^{44}$

En ce qui concerne la scène VII de l'acte III, nous pouvons constater que Gohier double le nombre de ses vers (passant de 41.5 à 92.5) et que les supressions qu'il effectue n'altèrent en rien ni les personnages ni l'intrigue. Ce qui n'est pas le cas avec la scène suivante.

Des 86 vers qui composent le long discours d'Antoine, discours que les Romains avaient ponctué de brèves réactions, il ne reste, en effet, chez Gohier que 15 vers. La scène VIII est ainsi diminuée de moitié passant de 100 à 48 vers. ${ }^{45}$

Privé de son discours, Antoine ne peut déployer son éloquence et marquer les étapes nécessaires qui persuaderaient son auditoire, il n'a plus à sa disposition que l'énoncé neutre de quelques évidences et un mol appel au soulèvement. Il est mis hors de combat dès le début de la scène, et les indications scéniques ne lui donnent aucun moyen de se faire valoir: plus de tribune aux harangues, plus de gestes mélodramatiques (Antoine à genoux auprès du cadavre de César).

Ces modifications entraînent nécessairement un résultat diamétralement opposé à celui de Voltaire: Antoine est vaincu et arrêté non seulement pour être un ami de César mais de plus pour vouloir lui succéder (César aspirant au titre de roi).

Les changements importants que Gohier a apportés à la scène VIII s'expliquent facilement.D'une part la leçon de Voltaire montrant un Antoine qui réussissait par son éloquence à retourner complètement le peuple est dangereuse en 1793 pour des raisons politiques évidentes. En effet, l'éloquence d'Antoine pouvait avoir un effet d'entraînement dans la salle où les spectateurs auraient pu se rendre à ses raisons et accepter sa conclusion à savoir qu'il faut pardonner au dictateur qui aime son peuple et châtier ceux qui osent attenter à ses jours quel que soit le but recherché. Or, cette mention de l'amour quasi paternel que César vouait aux Romains pouvait être interprété comme un rappel inopportun des thèmes de la propagande royaliste des premiers temps de la Révolution. Celle-ci alors n'hésitait pas à comparer Louis XVI à un bon père de famille entouré de ses enfants. Or, il faut se souvenir que lorsque Gohier écrit, la royauté a été abolie le 21 septembre 1792 et que le roi a été guillotiné le 21 janvier 1793: c'est en novembre de cette année-là que Gohier fait représenter sa version de La mort de César.

D'autre part, un discours modéré, comme celui d'Antoine, qui justifie une forme de gouvernement par l'existence de liens sensibles entre gouvernants et gouvernés risquait en 1793 d'être considéré comme contre-révolutionnaire d'autant plus que en 1793 comme en 1794, le 
théâtre fait constamment appel à une sensibilité républicaine, ${ }^{46}$ exploite la corde sensible des spectateurs par le spectacle attendrissant des liens filiaux, et conjugaux: ${ }^{47}$ la dévotion à la République-Mère avait remplacé la dévotion au Roi-Père.

De plus le spectacle de la manipulation politique sur scène et de sa réussite $a$, il faut y revenir, un effet fâcheux sur le peuple présent dans la salle: rappelons que les spectateurs pendant la Révolution ne sont plus les mêmes, ne serait-ce qu'à cause de la gratuité de certaines pièces représentées trois fois par semaine ${ }^{48}$ Compte tenu des circonstances et du besoin que les Jacobins ont alors de l'appui populaire, il n'est pas question de montrer sur scène combien le peuple est facile à manipuler, versatile dans ses opinions et capable de châtier ceux qu'il vient d'approuver. Ceci correspondait aux idées que Voltaire avait du peuple, ${ }^{49}$ Gohier, bien évidemment, ne peut les partager.

Enfin, et là il faut se référer à l'attitude de l'auteur, quand Voltaire nous montre Antoine condamnant les meurtriers de César, il faut comprendre qu'il condamne par là les tentatives de mise en place du régime républicain. Or, pour Gohier, il importe moins de condamner les meurtriers (qui ne le sont plus par un tour de passe-passe linguistique) que d'exalter le triomphe de la république, laquelle a été officiellement proclamée un an auparavant en France. Il est désormais important que la scène véhicule le même message que la tribune politique (cf. la demande du député Delacroix mise en épigraphe du présent texte).

Nous ne nous prononcerons pas sur la qualité des vers de Gohier en prenant pour acquis qu'un jugement de type esthétique $n^{\prime}$ a pas sa place dans le contexte de l'art révolutionnaire. La collaboration de Gohier à l'oeuvre de Voltaire a une valeur idéologique avant tout. Il utilise le théâtre comme un des moyens de propagande que la Révolution a connu et avant elle, le siècle des Lumières. Faut-il rappeler que pour la plupart des philosophes, la littérature était avant tout un outil de combat, un moyen de propager les Lumières vers ceux qui en avaient été privés? ? $^{50}$

La mort de César selon Gohier ne tint l'affiche que pour une douzaine de représentations, de novembre 1793 à juillet 1794, sur les théâtres parisiens. Le texte de Voltaire fut intégralement repris après le 9 thermidor an II: le discours d'Antoine fut de nouveau applaudi tandis que Brutus et ses complices se firent huer.

Quoi qu'il en soit, on peut observer que la Révolution n'a pas empêché cette pièce d'être représentée - même modifiée - pour un public qui, à cause du prix des places, ne l'aurait peut-être jamais découverte. Sachant que c'est en 1793 que la pièce connut le plus grand nombre de représentations (16), peut-on attribuer une partie de son succès aux modifications de Gohier? ou bien doit-on plutôt évoquer l'apport des subventions gouvernementales au théâtre républicain? Toujours est-il 
que l'abandon de la variante de Gohier n'a pas donné un regain de popularité à la pièce qui a vu ses représentations diminuer en 1794, 95, 96 pour cesser complètement en 1797 et 98.

Si l'intervention de Gohier peut être qualifiée de 'vandale,' de 'destructrice,' elle peut aussi être jugée 'conservatrice' et s'inscrire dans le double mouvement de destruction et conservation du patrimoine qui caractérisait la politique du gouvernement révolutionnaire: les mesures de protection et de sauvegarde du patrimoine intervenaient en même temps que celles qui visaient à le détruire. ${ }^{51}$

Les modifications apportées à La mort de César de Voltaire n'ont pas seulement permis à un nouveau public de la découvrir, elles ont encore contribué à assurer sa pérènnité.

\section{MURIEL USANDIVARAS-MILI}

Université Laurentienne

\section{Notes}

1 Cf. Colloque international de Clermont-Ferrand II, Révolution française et 'vandalisme révolutionnaire,' 15-17 décembre 1988.

2 Le Moniteur, t. XIII, no 226, 13 août 1792, page 398.

3 On peut citer la réflexion de deux orateurs du Faubourg St-Antoine dans un théâtre de Chartres vers la fin de novembre 1792: 'Nous avons renversé les rois et nous renverserons aisément les rois de théâtre' (Cité par Hérissay, la patriotisme dans le théâtre sérieux de la révolution, page 122).

4 Mentionnons le décret de la Commune de Paris du 21 août 1792 qui remplaça 'monsieur' par 'citoyen' et 'madame' par 'citoyenne.'

5 Toutefois, l'auteur pouvait les utiliser en les faisant suivre de l'expression 'vieux style' qui indiquait qu'il faisait référence à une ancienne manière de parler qui n'était plus en vigueur.

'Nous ne demandons pas qu' un homme ci-devant riche, ci-devant procureur, ci-devant comme il faut (vieux style), égoïste, célibataire et ruiné comme il le dit par la liberté, nous ne demandons pas qu'il nous aime' (Desprez, L'allarmiste, impromptu républicain en 3 actes et en prose, représenté le 3 thermidor an II).

6 Citons par exemple, Le COUSIN JACQUES qui est en réalité Louis Abel Beffroy de Regny, MONVEL qui recouvre Jacques Marie Boutet de Monvel, PIGAULT-LEBRUN, qui est en fait Antoine-Guillaume Pigault de l'Epinay.

7 Voir à ce sujet l'article de Antonio SERGI, 'Phèdre corrigée sous la Révolution,' Dix-huitième siècle, 1974, pp. 153-65.

8 La lecture de l'article de Sergi nous montre que l'essentiel des changements opérés sur Phèdre de Racine est d'ordre terminologique. Par exemple, les mots 'roi' et 'reine' sont remplacés par le prénom des personnages correspondants (Phèdre, Thesée) ou par leur état-civil (père, époux, épouse) ou par une rime dans 
le cas où ils se trouvent en fin de vers ('roi'='foi', 'reine'='peine'); d'autres mots trouveront des équivalents sémantiques acceptables, ainsi, 'vertu' mis pour 'sceptre,' 'foyer' pour 'trône.'

9 La première mention de ce changement est signalée dans Etienne et Martainville, Histoire du Théàtre-Français depuis le commencement jusqu'à la réunion générale (Paris 1802), 'comment souffrir que La mort de César fut souillée par le discours contre-révolutionnaire [ce mot est en italiques dans le texte] de ce modéré [id] $\mathrm{d}$ 'Antoine. Gohier se chargea de mettre Voltaire au pas [id] et refit tout le dénouement de La mort de César. Gohier fut successivement ministre de la Justice, président du tribunal criminel du département de la Seine, et membre du Directoire. Le dénouement de sa comédie politique ne valait pas mieux que celui qu'il avait fait pour La mort de César' (Etienne et Martainville, Histoire du Théatre-Français..., tome III, pp. 141-44). Ces auteurs, peu suspects de sympathie pour le théâtre de la Révolution, mentionnent également les retouches effectuées au Tartuffe de Molière et au Mahomet de Voltaire.

10 Voici en particulier le nombre de représentations de 1789 à 1799:

$1789: 0$

$1790: 3$

$1791: 1$

$1792: 3$

$1793: 16$

$1794: 8$

$1795: 9$

$1796: 7$

$1797: 0$

$1798: 0$

$1799: 3$

(ces chiffres nous ont été aimablement fournis par le professeur Emmet Kennedy de l'Université Georges Washington).

11 Préface de Voltaire à La mort de César, édition de 1736.

12 Discours sur la Tragédie, A Milord Bolingbroke. 1731.

13 Voltaire, les Lettres philosophiques, présentées par René Pomeau. (GF/Flammarion, 1964), p. 123.

14 Avertissement de l'édition Garnier des Oeuvres complètes de Voltaire, 1877, en tête de La mort de César.

15 Avertissement des éditions Kehl.

16 Le titre exact est Lettre de M. Algarotti à M. l'Abbé Franchini sur la tragédie de Jules César par M. de Voltaire. Elle est en italien et accompagnée d'une traduction.

17 ibid.

18 In Correspondance ou Oeuvres complètes de Voltaire, édition Moland, XXXIV, 49. Cité par Henri LION, les Tragédies et les théories dramatiques de Voltaire (Slatkine reprints Genève, 1970, réimpression de l'édition de Paris, 1895).

19 ibid.

20 ibid.

21 Voltaire, op. cit., p. 123.

22 A propos du secret de la naissance de Brutus, M. de Lamarre écrivait à Voltaire:'... secret dont la découverte produit le combat de la nature et de la patrie' (c'est nous qui soulignons). 
23 Louis Gohier, 1746-1830.

24 Voltaire, La mort de César, acte III, scène VI.

25 ibid, scène VII.

26 Un vers de Voltaire indiquait cet assentiment quand Cassius disait: 'Mais vous m'applaudissez, vous aimez tous la gloire' (dernier vers de la troisième tirade de Cassius, scène VII). Ce vers n'a pas été repris par Gohier.

27 Sur ce mot de 'roi' disons que Gohier n'hésite pas à utiliser dans cette scène (et même après) des mots qui ont été retranchés du vocabulaire républicain, tels que 'roi,' 'trône,' 'sceptre.' Il est vrai qu'en contrepartie il utilise le tutoiement républicain et que ce mot de roi a pour synonyme le mot 'brigand':

'De tes rois trop vantés, le meilleur est un maître / Voilà pour le brigand qui prétendrait à l'être' (Gohier, scène VII, III), dit Decime montrant son poignard. Voir à ce sujet les corrections apportées à Phèdre de Racine in Sergi, Phèdre corrigée....

28 Notons quand même que Voltaire avait mis dans la bouche de Cassius cette parole: 'J'ai tué mon ami pour le salut de Rome' (scène VII, III, La mort de César).

29 Ces deux vers seront repris par Gohier dans sa scène IX avec toutefois une modification pour le premier qui deviendra: 'Souvenez-vous toujours de ces serments sacrés.'

30 Scène VII, III de La mort de César, de Gohier, tirade 4.

31 ibid.

32 Voltaire, op.cit., scène VIII, acte III.

33 ibid.

34 ibid.

35 Ce dernier vers est la réplique presqu'exacte de celui prononcé par un Romain avant le discours d'Antoine: 'Oui, nous approuvons tous, Cassius et Brutus' (scène VIII, acte III, Voltaire).

36 Gohier, op. cit., scène VIII, acte III.

37 ibid.

38 En fait Gohier ne fait que reprendre et développer le dernier vers dit par Antoine dans le dénouement de Voltaire:

'Succédons à César en courant le venger' (La mort de César, VIII, III)

39 Gohier, op. cit., scène IX, acte III.

40 ibid.

41 À la fin de cette scène dans l'édition que nous avons utilisée, se trouve une note de bas de page qui dit: 'Le fond du théâtre s'ouvrait alors. On voyait la statue de la Liberté entourée d'un cercle de peuple. Dans la salle, tout le monde se levait, parterre et loges (G.A.).'

42 Gohier, op. cit., scène $X$, acte III.

43 ibid.

44 Sergi conclut du 'toilettage' de Phèdre qu'on ne peut 'parler d'un remaniement ou $\mathrm{d}^{\prime}$ une réécriture du texte' avec seulement $6 \%$ de modifications (retouches et supressions comprises). 
45 Il est curieux de constater que Gohier, pour les scènes VII et VIII, arrive presque au même nombre de vers que Voltaire: Voltaire : $41.5+100=141.5$; Gohier : 92.5 $+48=140.5$.

46 Voir à ce sujet $\mathrm{P}$. Trahard, Les Maîtres de la sensibilité révolutionnaire 1789-1794 (Boivin, 1936).

47 Au sujet des thèmes et de la fonction du répertoire théâtral pendant la Révolution française, voir notre thèse: M. Mili, Art politisé et politique de l'art: la problématique du phénomène théâtral en France pendant la Révolution 1789-1795 (Université Paris I-Sorbonne, 1974), exemplaire dactylographié.

48 Ce fut le cas notamment de Guillaume Tell de Sedaine, Caïus Gracchus de M-J. Chénier et Brutus de Voltaire.

49 Cette opinion est déjà nettement visible dans les endroits de La mort de César où Voltaire parle du peuple. En voici quelques échantillons:

'Je sais quel est le peuple: on le change en un jour;

Il prodigue aisément sa haine et son amour...

Il faut ...

Flatter encor ce tigre à l'instant qu' on l'enchaine,'

C'est César qui parle ainsi dans la scène IV de l'acte I.

'Ce peuple mou, volage et facile à fléchir,

Ne sait s'il doit encor l'aimer ou le haï'

$C^{\prime}$ est Brutus qui parle ainsi dans la scène IV de l'acte II.

'Précipitons ce peuple inconstant et facile:'

C'est Antoine qui parle dans la scène VIII de l'acte III.

Voltaire n'a pas varié sur l'opinion qu'il avait du peuple, il suffit de consulter le relevé statistique du mot PEUPLE fait par G. Mailhos dans les ouvrages historiques et la correspondance de Voltaire pour s'en assurer (voir G. Mailhos, Voltaire témoin de son temps, 1985, Aux amateurs de livres, atelier national, reproduction de thèses de l'Université de Lille III).

50 Voir à ce sujet $\mathrm{R}$. Niklaus, 'La propagande philosophique au théâtre au siècle des lumières,' Studies on Voltaire and the eighteenth century, vol XXVI (1963).

51 'Le despotisme en expirant a laissé à la France régénérée un superbe et vaste héritage qu'elle ne sauroit répudier sans honte...l'immense dépot de toutes les connaissances humaines, le résultat de tous les talens de l'esprit, le produit de toutes les citations du génie...il faut que le despotisme dont l'orgueil encourageoit et protégeoit les arts qui devoient enseigner à le détruire, soit à cet égard surpassé par la liberté': Boissy d'Anglas, cité par Edouard Pommier, en 'Idéologie et Musée à l'époque révolutionnaire,' Les images de la Révolution française, Actes du Colloque des 25-26-27 octobre 1985 tenu en Sorbonne (Publications de la Sorbonne, 1988), p. 65. Tout l'article de Pommier pourrait être cité pour illustrer cette 'valse-hésitation' entre le désir de destruction et de conservation durant la Révolution française. 